Journal of Biomedical and Pharmaceutical Research

Available Online at www.jbpr.in

CODEN: - JBPRAU (Source: - American Chemical Society)

NLM (National Library of Medicine): ID: (101671502)

Index Copernicus Value 2018: 88.52

Review Article

Volume 10, Issue 4: July-August : 2021, 01-06

ISSN (Online): 2279-0594

ISSN (Print): 2589-8752

\title{
Free Radicals, Reactive Oxygen Species \& Antioxidants in Periodontitis - A Review
}

\author{
Dr. Sankar Narayana Sarma ${ }^{1}$, Dr. Nalli Prasanth Kumar ${ }^{2}$, Dr. Haritha \\ Sarayavalasa $^{3}$, Dr. Venkata Ratnakar Guduri ${ }^{4}$ \\ ${ }^{1}$ Senior Lecturer Department of Periodontology GSL Dental College \& Hospital Rajahmundry \\ Andhra Pradesh, India \\ ${ }^{2}$ Senior Lecturer, Department of Oral Medicine and Radiology, Lenora Institute of Dental \\ Sciences, Rajahmundry, Andhra Pradesh, India \\ ${ }^{3}$ DENTAL Assistant Surgeon, Govt. of Andhrapradesh CHC Kapileswarapuram East Godavari \\ District Andhrapradesh \\ ${ }^{4}$ Senior Lecturer Department of Periodontology GSL Dental College \& Hospital Rajahmundry \\ Andhra Pradesh, India
}

Article Info: Received 20 June 2021; Accepted 29 July 2021

DOI: https://doi.org/10.32553/jbpr.v10i4.871

Corresponding author: Dr. Sankar Narayana Sarma

Conflict of interest statement: No conflict of interest

\begin{abstract}
Periodontal disease is a chronic inflammatory disorder that leads to tissue damage and bone loss. Free radicals have been implicated in the pathogenesis of periodontal disease.Oxidative stress is considered to be the heart of periodontal tissue damage that results from host- microbial interactions.Various studies shows that antioxidant therapy plays a crucial role in the treatment of periodontitis.
\end{abstract}

\section{Introduction}

Periodontal disease is a chronic inflammatory disorder that leads to tissue damage and bone loss as a result of the complex interaction between pathogenic bacteria and the host's immune response. Free radicals have been implicated in the pathogenesis of periodontal disease $^{1,2}$.

Oxidative stress is considered to be the heart of periodontal tissue damage that results from host- microbial interactions. ${ }^{3}$ To protect the cells and organ systems of the body against reactive oxygen species, humans have evolved a highly complex antioxidant protection system thatfunction interactively and synergistically to neutralize free radicals. Thus the studies shows that antioxidant therapy plays a crucial role in the treatment of periodontitis as the Oxidative stress is considered to be the heart of periodontal tissue damage that results from host-microbial interactions ${ }^{3}$

General Review

Free Radical (FR) and Reactive Oxygen Species (ROS):

Nomenclature, Features, Classification, and Sources. Free Radical: 
A free radical is any species capable of independent existence which contains one or more than one unpaired electrons. The simplest free radical is an atom of the element hydrogen, with one proton and a single electron. Free radicals can be generated in a wide variety of chemical and biological systems. ${ }^{2}$

There are three possible means of FR formation 4

(A) By the homolytic cleavage of the covalent bond of a normal molecule, (i.e., homolyticfission),

(B) By the loss of a single electron from a normal moleculeA + B: $\rightarrow$ A- + B.+ ("-" and/or "+" charged FR)

$\mathrm{A}: \mathrm{B} \rightarrow \mathrm{A}+\mathrm{B}$ (electrically neutral FR)

(C) By the addition of a single electron to a normal molecule, called as "electron transfer". In recent years, the term reactive oxygen species (ROS) has replaced the term free radicals as it's a more global term. ${ }^{4}$

\section{Reactive Oxygen Species:}

Reactive oxygen species (ROS) is a term that encompasses all highly reactive, oxygencontaining molecules, including free radicals. Types of ROS include the hydroxyl radical, the super-oxide anion radical, hydrogen peroxide, singlet oxygen, and various lipid peroxides. All are capable of reacting with membrane lipids, proteins and enzymes, and other small molecules, resulting in cellular damage. ${ }^{5}$

Sources of Reactive Oxygen Species. ${ }^{6}$

\section{Exogenous Source:}

Heat, trauma, ultrasound, ultraviolet light, ozone, smoking, exhaust fumes, radiation, infection, excessive exercise, and therapeutic drugs.

\section{Endogenous Source:}

Results in formation of ROS include:-

1. Bi-products of metabolic pathways: During cell metabolism, electrons leak from their transporters at a constant rate during the process of glycolysis, reducing oxygen to super-oxide anion.

2. ROS can also be generated by phagocytes and cells of the connective tissue. The process comprises the "respiratory burst" and is stimulated by a variety of mitogens or antigens.

\section{Targets for FR \& ROS}

There are five principal targets for FR and ROS in living cells: ${ }^{6,7}$

(1) Small organic bio molecules,

(2) Proteins,

(3) Nucleic acids,

(4) Gene activation, and

(5) Unsaturated fatty acids

\section{Potential Mechanisms for Periodontal Tissue Destruction By $\operatorname{ROS}^{3}$}

Whilst most ROS have extremely short halflives as ${ }_{10}-9$ to $10^{-} 6$, they can cause substantial tissue damage by initiating free radical chain reactions. Different mechanisms, which mediate tissue damage, into two types ${ }^{3,4}$

\section{Direct Tissue Damage:}

Protein damage including gingival hyaluronic acid and proteoglycansI

Lipid per oxidation (through activation of cyclooxygenases and lipoxygenases).

DNA damage.

\section{Indirect Tissue Damage}

Oxidation of important enzymes e.g. antiproteases such as $\alpha$-1 antitrypsin.

Stimulation of pro-inflammatory cytokines release by monocyte $\mathrm{s}$ and macrophages by depleting intracellular thiol compounds and activating nuclear factor $\mathrm{kB}$

(NFkB)

Fig:1 Simplified diagram illustrating a central role of ROS in generating chronic inflammation and tissue damage in response to periodontal pathogens. 


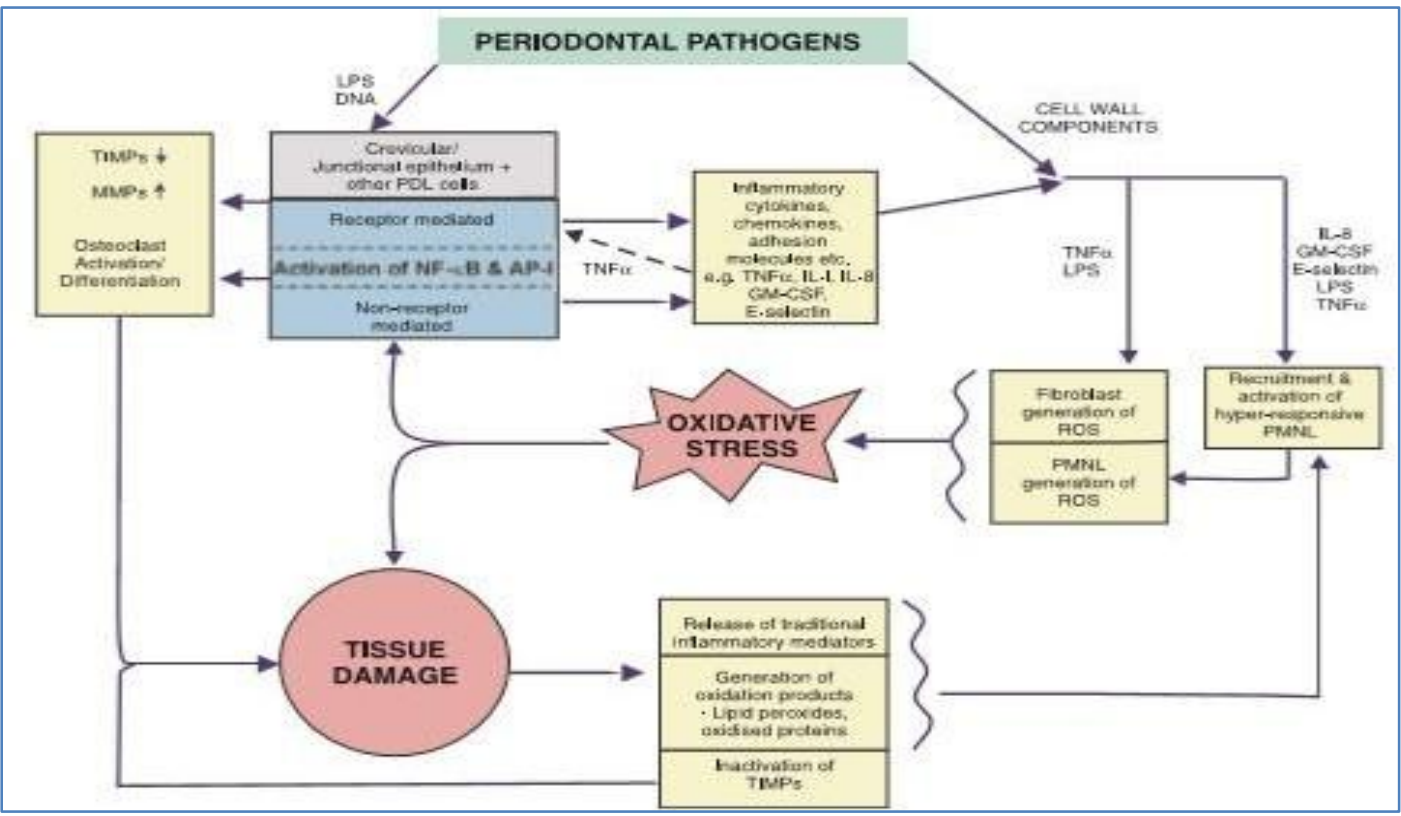

Figure 1:

\section{Effects of ROS on periodontal tissues and components:}

The reactive oxygen species cause periodontal tissue damage by,

\section{Ground substance degradation}

2. Collagenolysis either directly or indirectly or as a result of oxidation of pro teases

3. Stimulation of excessive pro-inflammatory cytokines release through $\mathrm{NF}-\kappa \mathrm{B}$ activation.

4. PG-E2 production via lipid per oxidation and super-oxide release, both of which have been linked with bone resorption.

5. Since IL-1 \& TNF- $\alpha$ positively regulate their own production, the additive effects of endotoxin mediated cytokines production and that arising from the respiratory burst of PMNLsin response to the same organisms, lead to periodontal inflammation and

6. subsequent attachment loss ${ }^{8}$

Effects of ROS on periodontal tissues and components occur in ${ }^{9}$

1. Gingival cells

2. Bone resorption

3. Ground substance
4. Collagen

5. DNA

\section{Role of Anti-Oxidants in Periodontitis:}

Antioxidant control free radical damage at the cellular level not only to premature in periodontal disease. Antioxidants neutralize free radicals by donating an electron without becoming unstable themselves. Researchers assessed levels of vitamins $\mathrm{A}, \mathrm{C}$ and $\mathrm{E}$, selenium, a-carotene, b-carotene lycopene and lutein in periodontal disease status. ${ }^{10}$

\section{Antioxidant Defense:}

Antioxidant defense system (ADS) against oxidative stress is composed of several lines and antioxidants are classified into four categories based on their function.

- First: - Preventive antioxidants which suppress formation of free radicals.

- Second: - Radical scavenging antioxidants which suppress chain initiation and breaking chainpropagation reactions.

- Third: - Repair and denovo antioxidants.

- Fourth: - Adaption where the signal for the production and actions of free radicals 
induces formation and transport of the appropriate antioxidant to the right site. ${ }^{11}$

\section{Classification of Antioxidants: ${ }^{3,6}$}

Antioxidants can be categorized by several methods:

\section{Classification of antioxidants by chemistry}

Antioxidants are grouped into two namely;

(1) Primary or natural antioxidants.

(2) Secondary or synthetic antioxidants.

\section{Primary or natural antioxidants}

They are the chain breaking antioxidants which react with lipid radicals and convert them into more stable products. Antioxidants of this group are mainly phenolic in structures and include the following

(a) Antioxidants minerals - These are co factor of antioxidants enzymes. Their absence will definitely affect metabolism of many macro molecules such as carbohydrates. Examples Include selenium, copper, iron, zinc and manganese.

(b) Antioxidants vitamins - It is needed for most body metabolic functions. They include vitamin $\mathrm{C}$, vitamin $\mathrm{E}$, vitamin $\mathrm{B}$.

(c )Phytochemicals- These are phenolic compounds that are neither vitamins nor minerals.

\section{Secondary or synthetic antioxidants}

These are phenolic compounds that perform the function of capturing free radicals and stoppingthe chain reactions and the compounds include

- Butylated hydroxyl anisole (BHA)

- Butylated hydroxyrotoluene (BHT)

- Propyl gallate (PG) and metal chelating agent (EDTA)

- Tertiary butyl hydroquinone (TBHQ)

- Nor dihydroguaretic acid (NDGA)

\section{The Antioxidant Process:}

Antioxidants block process of oxidation by neutralizing free radicals. In doing so, antioxidantsthemselves become oxidized.

Antioxidants act in two ways as

Chain-breaking

Preventive

\section{Chain breaking Antioxidants:}

When a free radical releases or steals an electron, a second radical is formed. This molecule then turns around and does same thing to a third molecule, continuing to generate more unstable products. The process continues until termination occurs either radical is stabilized by a chain- breaking antioxidant such as beta carotene, vitamin $\mathrm{C}$ and vitamin $\mathrm{E}$.

\section{Preventive}

As the first line of defense against formation of ROS is prevention, which occurs by enzymatic antioxidants like super-oxide dismutase, catalase and glutathione peroxidase enzymatic antioxidants prevent oxidation by reducing rate of chain initiation. They can also prevent and oxidation by stabilizing transition metal radicals such as copper and iron. ${ }^{12}$

\section{super-oxide Dismutase (SOD):}

super-oxide Dismutase is an Endogenous Enzymatic antioxidant, it is the first enzyme involvedin the antioxidant defense. super-oxide dismutase is a metalloprotein found in both prokaryotic and eukaryotic cells. ${ }^{11}$ super-oxide (O2) is formed chemically by addition of an extra electron to the oxygen molecule. superoxide is regarded as a weakly reactive radical, relative to the hydroxyl radical, but can attack and cause cell damage. super-oxide is removed from tissues by spontaneous dis mutation to hydrogen peroxide, a process that can also be catalyzed by an enzyme called super-oxide dismutase.SOD enzymes are largely found both intracellularly and extracellular environment. ${ }^{1}$ The different type of SOD exist Depending on metal ion content the SOD is grouped as 
- $\mathrm{Cu} / \mathrm{Zn} \mathrm{SOD}$

- Mn SOD

- Fe SOD Antioxidants.

The different types of SOD are differently distributed among organisms as well as among sub cellular compartments, and they contain metals (copper, zinc, manganese, or iron) essential for their catalytic function. ${ }^{7}$

Factors to account for when contemplating antioxidant approaches to therapy ${ }^{3}$

When considering antioxidant approaches to the management of periodontal diseases a number of factors must be considered:

1. There should be evidence of excess ROS production associated with the presence of disease(ideally locally to the diseased tissues);

2. There should be evidence of ROS-mediated tissue damage either by:

(a) Direct effects of ROS activity (biomarkers) measured locally;

1. Indirect effects of ROS activity via hyper inflammation as a result of local redox sensitive transcription factor activity and subsequent imbalances of pro- and anti inflammatory cytokines

2. There should be clear mechanistic links between oxidative stress, the observed tissue damage and the mode of activity of the antioxidant.

3. Supplementation with the antioxidant should reduce the incidence of disease at the affectedsite or tissues.

4. Supplementation with the antioxidant should reduce disease recurrence;

5. Subjects with the disease should have a demonstrable local deficiency of the antioxidant, or oftotal antioxidant capacity;

6. Subjects without disease should have no antioxidant deficiency;

7. Restoration of the antioxidant level locally should improve clinical measures of disease.
8. Adjunctive use of the anti oxidant with traditional therapies should provide improved treatment outcomes over non-surgical therapy alone.

9. Markers of local ROS activity should decrease with antioxidant therapy.

(b) To consider prophylactic use of antioxidant in periodontitis patient's criteria $1-5$ should be satisfied. To consider use of antioxidants in active periodontal therapy criteria 5-10 should be satisfied.

\section{SUMMARY \& CONCLUSION}

(c) Tissue injury in human disease is accompanied by an imbalance in the oxidant/ antioxidant status, creating oxidative stress. ${ }^{2}$ Oxidative stress lies at the heart of the periodontal tissue damage that results from host- microbial interactions, either as a direct result of excess ROS activity/antioxidant deficiency or indirectly as a result of the activation of redox-sensitive transcription factors and the creation of a pro-inflammatory state. ${ }^{3}$ Free radicals and other reactive oxygen and nitrogen species are generated in vivo, and their generation is continuous process. Antioxidants can play very important role. Their levels are modulated by a network of antioxidant defence systems, assisted by repair systems. ${ }^{2}$ The reaction of the hosts inflammatory and immune responses to pathogenic species within the periodontal environment can generate reactive oxygen species within the adjacent tissues. Protection against such tissues is provided byantioxidants, many of which are released locally at sites of infection, by PMNL's themselves and other tissues such as epithelium. ${ }^{1}$ FR/ROS are essential to many normal biological processes, and low doses of certain radicals or radicalderived species can stimulate the growth of fibro-blasts and epithelial cells in culture. When anti-oxidant systems are unable to counteract their action efficiently, tissue damage can result. There is evidence showing that similar effect may take place in Periodontitis as in other inflammatory diseases. 
This concept has led to a search for appropriate "antioxidant therapy" in inflammatory disease. The successful therapeutic manipulation of the cellular response by antioxidant molecules might necessitate the maintenance of the critical balance between FR/ROS and antioxidant defense systems. It may benecessary to deliver antioxidants selectively to specific cell types and to define the concentrations suitable for blocking inappropriate cell responses but leaving the unimpaired physiological levels of FR/ROS activity necessary for normal cell function. ${ }^{6}$ The best recommended action is to increase the intake of natural antioxidant vitamins by consuming cereals, pulses, nuts, fruits, vegetables, which seems to be a safe approach $^{6}$ The resulting increased oxidative damage to bio molecules may play an important role in the pathophysiology of periodontal disease and is amenable to therapeutic intervention with appropriate antioxidants $^{2}$

\section{Bibliography}

1. Chapple ILC: Reactive oxygen species and antioxidants in inflammatory diseases. J Clin Periodontol 1997: 24: 287-296.

2. Barry Halliwell Free Radicals and other reactive species in Disease encyclopedia of life sciences / \& 2001 Nature Publishing Group

3. Chapple \& john b. Matthews. The role of reactive oxygen and antioxidant species in periodontal tissue destruction
Periodontology 2000, Vol. 43, 2007, 160-232.

4. ILC Chapple Oxidative stress, nutrition and neutrogenomics in periodontal health and disease Int J Dent Hygiene 4 (Suppl. 1), 2006; 15-21

5. DR. Mark Pervical Antioxidants Clinical Nutrition Insights NUT 031 1/96 Rev. 10/98

6. M. Battino, P. Bullon, M. Wilson, and $\mathrm{H}$. Newman oxidative injury and inflammatory periodontal diseases: the challenge of anti-oxidants to free radicals and reactive oxygen species Crit Rev Oral Biol Med 10(4):458-476 (1999)

7. Alok Sharma, Swati Sharma Reactive Oxygen Species and Antioxidants in Periodontics: A Review international journal of dental clinics 2011:3(2):44-47

8. Roger T. DEAN and Michael J. DAVIES Biochemistry and pathology of radicalmediated protein oxidation Biochem. J. (1997) 324, 1-18

10. Forman HJ, Fukuto JM, Torres Redox signaling: thiol chemistry defines which reactive oxygen and nitrogen species can act as second messengers. Am J Physiol Cell

11. Physiol. 2004 Aug; 287(2):C246-56.

12. Toby Lawrence The Nuclear Factor NF$\kappa B$ Pathway in Inflammation Cold Spring Harb Perspect Biol. 2009 Dec; 1(6): a001651. 